\title{
Associations Between Mildly Impaired Autistic Boys' and Girls' Challenging Behaviour and Parental Anxiety and Depression
}

\author{
Vicki Bitsika $^{1} \cdot$ Christopher F. Sharpley $^{1,2}$ (D)
}

Accepted: 21 December 2021 / Published online: 24 January 2022

(C) The Author(s) 2022

\begin{abstract}
To investigate the contribution made by autistic children's Challenging Behaviour (CB) to their parents' anxiety and depression, parents of 32 autistic boys and 32 autistic girls (aged 6 to 18 years) completed standardised instruments on their children's behaviour and their own anxiety and depression. Significant associations were found between the children's CB and their parents' anxiety and depression, but the patterns of those associations differed according to the sex of the child. At the individual CB level, boys' change of mood, but girls' social isolation, were most powerfully associated with their parents' depression. Girls' impulsive behaviour was most strongly linked with their parents' anxiety.
\end{abstract}

Keywords Autism · Challenging behaviour · Parent · Anxiety $\cdot$ Depression

Parents of a child with Autism Spectrum Disorders (ASD) often suffer from an elevated prevalence of anxiety and depression (Bitsika \& Sharpley, 2004; Kuusikko et al., 2008; Mugno et al., 2007), significantly more so than parents of children with other disorders (Hayes \& Watson, 2013). As well as being intrinsically distressing, anxiety can be a precursor of low-level illnesses (Fries et al., 2005), or emotional and behavioural problems such as demoralization, hostility and mistrust (Langewitz $\&$ Ruddell, 1989), an increased risk of relationship problems and cognitive difficulties (Nutt, 2004), and elevated risk of suicide (Malone et al., 1995; Zimmerman et al., 2000). Depression has greater adverse effects on personal health (Moussavi et al., 2007) and higher costs of care (Langa et al., 2004) than other chronic diseases, is also associated with suicide in about $15 \%$ of all depressed patients (APA, 2013),

Christopher F. Sharpley

csharpl3@une.edu.au

1 Brain-Behaviour Research Group, University of New England, Armidale, NSW, Australia

2 School of Science \& Technology, University of New England, Queens Elizabeth Drive, Armidale, NSW, Australia 
and carries a similar risk for death as smoking, even when related health factors such as blood pressure, alcohol intake, cholesterol and social status are taken into account (Mykletun et al., 2009). All of these outcomes of anxiety and depression may impinge upon ability to interact and carry out family and carer tasks, which can be particularly demanding for parents of autistic children, who often find themselves unable to cope with their children's behaviour (Sharpley et al., 1997).

Research which focusses upon the possible 'contributors' to anxiety and depression in parents of autistic children might help in the development of strategies for avoiding these disorders. One possible contributor to parental anxiety and depression is the 'challenging' behaviour (CB) sometimes exhibited by these children. CB has been shown to cause parents great distress (Estes et al., 2009; Lecavalier et al., 2006), even more than the severity of the core ASD-related symptomatology itself, i.e., difficulties in social communication and social interaction, plus restricted or repetitive patterns of behaviour (APA, 2013). Although these core symptoms include autistic children's extreme distress reactions to variety in their environment, and adverse responses to sounds and textures, it is the additional behavioural outbursts such as hitting, kicking, screaming, breaking objects, running away, and extreme irritability which are most commonly the cause of parental stress (O'Nions et al., 2018).

However, previous studies of the association between child CB and parental anxiety and depression have used instruments with limited value for that purpose. For example, although the study by Falk et al. (2014) measured stress, anxiety and depression in parents by using the Short Form of the Stress, Anxiety, and Depression Scale (DASS) (Lovibond \& Lovibond, 1995), the authors of that scale state that it does not include all the diagnostic criteria for Generalised Anxiety Disorder (GAD) or Major Mood Disorder (MMD) as they are defined in the DSM-5 (APA, 2013). Much of the previous research in this field has used scales of 'parental stress' (e.g., Ashworth et al., 2019; Giovagnoli et al., 2015; Rao \& Beidel, 2009; Shawler \& Sullivan, 2017; Tomanik et al., 2004) rather than GAD and MDD. GAD and MDD can be measured via clinical interview, but parents' self-reports of their own anxiety and depression on standardised scales may offer a unique and personally-verified perspective on their mental health that is strongly correlated with clinicians' diagnoses (Kroenke et al., 2001; Spitzer et al., 2006). There are also some data suggesting that self-reports are more self-revealing of suicidal ideation than clinician interviews (Kaplan et al., 1994). Accessing parents' GAD and MDD status via self-report also ensures that these data are collected simultaneously as CB information, rather than (for example) from a clinical diagnosis received some time previously. Additionally, parents' own global estimates of their anxiety or depressive states without any reference to the actual diagnostic criteria for GAD or MDD provide only a limited dataset of the specific GAD and MDD symptomatology.

CB exhibited by autistic children is most often measured by parents' ratings of their child's behaviour on standardised scales because parents are the primary caregivers and often spend most time with their autistic child in home settings. Typical scales for measuring CB include a wide range of CBs (e.g., Aman et al., 1985). However, examination of the associations between total scores on such scales and parental anxiety and depression may not identify which types of CBs contribute 
most strongly to parental anxiety and depression, or even the individual CB itself, thereby limiting the clinical relevance of findings.

Although most research on autistic children has been conducted on samples of boys, recent attention to autistic girls adds to the understanding of the population of autistic children, and so a sample of young autistic males and females was recruited for this study, plus one of their parents. As well as representing both sexes, such data also allow for direct comparison of the $\mathrm{CB}$ of autistic girls and boys, the relative severity of their parents' anxiety and depression, and how the children's CBs are associated with their parents' anxiety and depression. Because this study is part of a larger investigation of anxiety and depression among parents of school-age autistic children (Bitsika \& Sharpley, 2016), the autistic children were recruited from those who were attending primary (elementary) and secondary school. Due to the limited use of appropriate instruments to measure parental GAD and MDD in previous research, this study aimed to collect that data using standardised self-report scales based upon the DSM-5 diagnostic criteria for those disorders, and to make comparisons between parents of autistic boys and autistic girls, previously unreported in the literature. Because the study was exploratory, no directional hypotheses were raised for testing.

\section{Method}

\section{Participants}

In response to an advertisement to local parent support groups of families with at least one autistic child on the Gold Coast, Australia, 64 parents and their autistic children (32 boys, 32 girls, all aged from 6 to 18 years), were recruited to this study. There is evidence of sex dysphoria in autistic children (Glidden et al., 2016), and so the 'boys' and 'girls' in this study were defined by their parents' identification of their sons' and daughters' sex at birth, plus the boys and girls themselves agreeing to participate in 'a study about autism in boys' or 'a study about autism in girls' (recruited separately). None of these boys or girls indicated in any way that they did not consider themselves to be the sex that their parents assigned them. All 32 parents of the autistic girls were mothers, and 28 of the autistic boys' parents were also mothers, although no restriction was placed on which parent could respond to the advertisement. Although both fathers and mothers may experience anxiety and depression arising from their autistic child's CB, it is mothers who have the highest levels of parenting stress from caring for a child with ASD (Dabrowska \& Pisula, 2010), and so this restriction of fathers' data was deemed acceptable at this stage of investigation. All of the autistic children had been diagnosed with ASD via clinical interviews with a paediatrician or psychiatrist, and a clinical psychologist. Those diagnoses were confirmed by administration of the Autism Diagnostic and Observation Schedule- $2^{\text {nd }}$ edition (ADOS-2) (Lord et al., 2012) by a research-reliable assistant as part of the recruitment process for this study; all the boys and girls had ADOS-2 Total scores of 7 or more. IQ was also obtained for each child via the Wechsler Abbreviated Scale for Intelligence- $2^{\text {nd }}$ edition (WASI-II) (Wechsler, 2011) administered by the same 
research-reliable assistant during recruitment; all boys and girls had Full Scale IQ scores of 70 or greater. None of the parents reported that they were currently taking medication for anxiety or depression or that their autistic children had been formally diagnosed with anxiety or depression. The autistic children were able to be classified as "mildly impaired" because of their IQ, ASD severity scores, their ability to selfmanage, and because they were all attending mainstream schools.

\section{Instruments}

\section{Depression and Anxiety}

Parental anxiety and depression were self-assessed via the seven-item GAD7 (Spitzer et al., 2006) and the nine-item Patient Health Questionnaire-9 (PHQ9) (Kroenke et al., 2001) respectively. These scales are based upon the diagnostic criteria for Generalised Anxiety Disorder (GAD) and Major Depressive Disorder (MDD) as they were described in the DSM-IV-TR (APA, 2000) and which remain consistent in the current DSM-5 (APA, 2013). The GAD7 items ask respondents to state how often they have been bothered by specific anxiety problems during the last two weeks, using a rating scale of "Not at all" (scored as 0), "Several days" (1), "More than half the days" (2) and "Nearly every day" (3). Total scores range from 0 to 21 , and the GAD has cutoff scores of: 0-4="minimal", 5-9="mild", 10-14="moderate" and 15-21="severe" anxiety (Spitzer et al., 2006). The GAD7 has specificity and sensitivity of 0.80 , with internal consistency (Cronbach alpha) of 0.92 and test-retest reliability of 0.83 over one week (Spitzer et al., 2006). The same self-rating scale is used for the PHQ9, with total scores ranging from 0 to 29; cutoff scores for MDD severity are: 1 to 4 (signifying a rating of "none"), 5 to 9 ("mild"), 10 to 14 ("moderate"), 15 to 19 ("moderately severe") and 20 to 27 ("severe") (Spitzer et al., 1999). The PHQ9 has specificity and sensitivity above $95 \%$, and internal consistency of 0.89 (Kroenke et al., 2001).

\section{Challenging Behaviour}

The autistic sons' and daughters' CB was assessed by their parents, using the Aberrant Behavior Checklist (ABC) (Aman et al., 1985), a 58-item scale for rating the behaviour of an individual on five subscales (Irritability, Lethargy, Stereotypy, Hyperactivity, Inappropriate Speech) that are relevant to ASD and which have internal consistencies (Cronbach's alpha) between 0.77 and 0.94 . Respondents are asked to provide ratings about the severity of each of the 58 items for a given individual. Ratings are given on a 4-point Likert scale ( $0=$ "not at all", $1=$ "slight", $2=$ "moderate", $3=$ "severe") for the individual's behaviour "during the last four weeks". The Community version of the $\mathrm{ABC}$ was used because all these autistic youth fitted that category. Normative data have been reported for ages 6 to 12 years from a sample of 1,893 young autistic people (Kaat et al., 2014). The ABC provides a total CB score, and individual scores for each of the five subscales described above. Because the number of $\mathrm{ABC}$ items used in these five subscales ranges from 4 to 16 , mean subscale scores were used. 


\section{Procedure}

Following their expression of intent to participate in the study, parents of the autistic children were given an Information Statement and Consent Form for themselves and their children, and a questionnaire booklet that included the PHQ9 and GAD7 to answer about themselves, and the ABC to answer about their children. These assessments were to be completed with the same four-week period for all participants, in order to reduce the influence of possible history effects. These questionnaires were collected by research staff and checked to ensure completeness.

\section{Statistical Analyses}

SPSS 25 was used to obtain descriptive data and check for normality. MANOVA detected any differences between male and female data. Pearson correlation coefficients were used to test for: significant associations between boys' and girls' age, IQ and ASD severity; children's ABC total and subscale scores and individual ABC items; and parents' GAD7 and PHQ9 scores. Hierarchical regression analyses identified the specific ABC items that contributed most to the variance in parents' GAD7 and PHQ9 scores. Appropriate corrections were made to the alpha levels in recognition of the likelihood of family-wise error rates from multiple comparisons, but the major statistical criterion was presence of a recognised effect size. For ANOVA and MANOVA, this was partial eta squared, and for correlational analyses the categories set by Cohen (1988, pp 79-81) of $r=0.10$ to 0.29 (small effect), $r=0.30$ to 0.49 (medium), and $r=0.50$ to 1.0 (large) were used.

\section{Results}

\section{Descriptive Data}

Table 1 presents the descriptive data for the autistic children's ages, WASI-II, ADOS-2, and ABC mean scores, plus their parents' GAD7 and PHQ9 scores, broken down according to the sex of the child. The very small number of fathers did not allow for the application of statistical comparisons on the basis of parent sex. There was little evidence of skewness or kurtosis in any of these scores, and inspection of the histograms and testing for normality indicated no need for transformation of the data (Tabachnik \& Fidell, 2013). Internal consistency for the GAD7 was 0.874 , and for the PHQ9 it was 0.841 , both of which are acceptable. There were no significant correlations between the autistic girls' age, IQ, or ASD severity and any of their ABC scores, but boys' age was significantly inversely associated with their ABC mean Irritability $(r=-0.500, p=0.004)$ and Hyperactivity $(r=-0.561, p=0.001)$ scores. Both of these associations were at the 'large' effect level and both indicated that younger autistic boys were more likely to exhibit irritability and hyperactivity than their older peers. Age was therefore covaried out of the following analyses of boys' data. There were no 


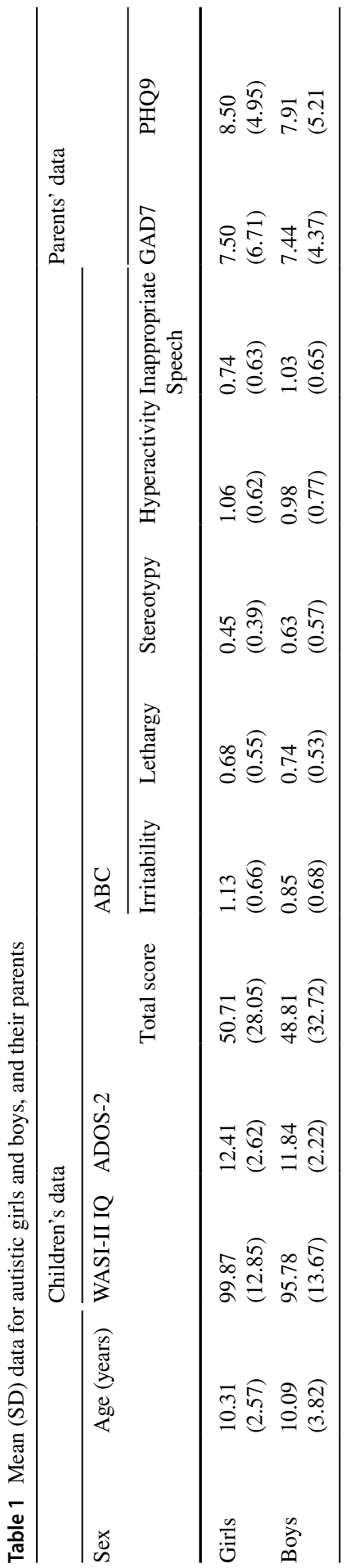


significant correlations between the autistic boys' IQ or their ASD severity and their ABC scores.

\section{Child Sex Differences}

MANOVA of the age, WASI-II IQ and ADOS-2 scores of the autistic boys and autistic girls revealed a nonsignificant main effect $F(3,60)=1.565, p=0.207, \mu^{2}=0.073$ ) and no significant univariate effects (all $p$ values $>0.100$ ). This allowed the autistic boys and girls to be compared for differences in ABC total and subscale scores free from the possible confound of age, IQ, or ASD severity. ANOVA indicated that there was no significant difference in the ABC total scores between autistic girls and boys $F(1,63)=0.062, p=0.804, \mu^{2}=0.001$. MANOVA on the ABC subscales mean scores showed no significant univariate effects for the $\mathrm{ABC}$ subscales (all $p$ values $>0.098$ ), indicating that the severity of $\mathrm{CB}$ was similar across the autistic boys and girls.

\section{Parental GAD7 and PHQ9}

\section{Anxiety, Depression, Severity and Sex of Child}

There were no significant differences in the GAD7 scores reported by the boys' parents compared to the girls' parents, $F(1,63)=0.003, p=0.954, \mu^{2}=0.001$, nor for the PHQ9 scores across the two groups of parents $F=0.218, p=0.642, \mu^{2}=0.004$, with sex of parent covaried out of the analysis due to the small number of fathers in the autistic girls' parent sample. The severity of the GAD7 and PHQ9 scores given by these parents was determined by reference to the norming data reported by the authors of the GAD7 and PHQ9 that recommended cutoff scores for severity (described in Methods). The relative distributions of those categories of GAD7 and PHQ9 scores are shown for the boys' and girls' parents in Fig. 1, plus the data from a recent study of 13,829 Australians recruited from the general community (Stocker et al., 2021) as a comparison source. Although there are some apparent differences in the frequency of these severity categories across the parents of boys and girls, those apparent differences did not reach statistical significance: GAD7 $\chi^{2}(3)=0.111$, $p=0.990$; PHQ9 $\chi^{2}(4)=5.371, p=0.251$. However, some cells were small, and so any conclusions regarding differences between parental groups should be treated with caution for the more severe levels of anxiety or depression.

\section{Associations With Child Background Variables}

There were no significant correlations between the autistic boys' age, IQ, or ASD severity and their parents' GAD7 or PHQ9 scores; nor were there any significant correlations between the autistic girls' age, IQ or ASD severity and their parents' GAD7 or PHQ9 scores (all $p$ values $>0.2$ ). 
(a)

\section{GAD7 scores}

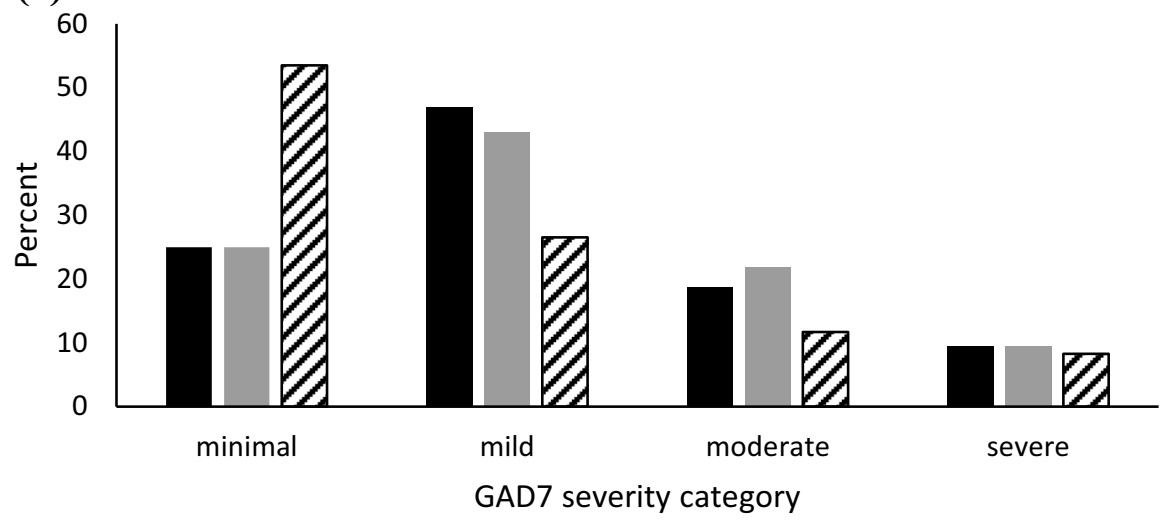

- Boys' parents Girls' parents $\square$ Stocker, et al.
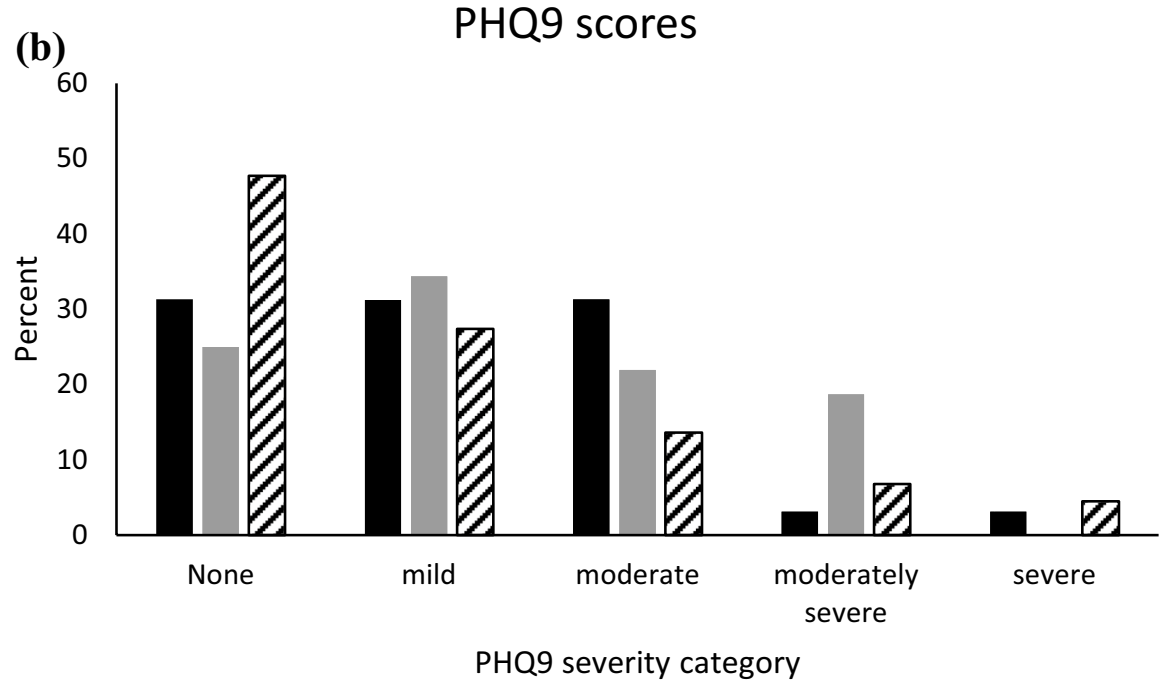

- Boys' parents Girls' parents $\mathbf{\square}$ Stocker, et al.

Fig. 1 (a) Parents' GAD7 score severity (b) Parents' PHQ9 score severity

\section{Child ABC and Parental GAD7 and PHQ9}

\section{Total ABC Scale Level}

Boys' ABC total score was significantly correlated with their parents' GAD7 $(r=0.359$, $p=0.047)$ and PHQ9 $(r=0.498, p=0.004)$ scores; similar results were found for the 
girls' parents' GAD7 $(r=0.449, p=0.010)$ and PHQ9 $(r=0.428, p=0.015)$ scores and the girls' ABC total scores.

\section{Subscale Score Level}

Table 2 presents the correlation coefficients between the five $\mathrm{ABC}$ subscales and parental GAD7 and PHQ9 scores for the boys and girls, with the coefficients that reached at least the upper range of 'medium' strength correlation (Cohen, 1988) shown in bold. It is apparent from these data that there were different patterns of association between the autistic girls' ABC scores and their parents' GAD7 and PHQ9 scores and those shown by the autistic boys and their parents. The boys' ABC Irritability score was significantly associated with their parents' GAD7 and PHQ9 scores, but that was not the case for the girls, for whom Lethargy appeared to be linked with their parents' PHQ9. Boys' Hyperactivity was significantly associated with their parents' PHQ9 scores, but girls' Hyperactivity was significantly associated with their parents' GAD7 scores.

Hierarchical regression was used to further explore these correlational data. The boys' Irritability scores made a significant contribution to their parents' PHQ9 scores (R squared change $=0.371, F(1,30)$ for change $=17.071, p=0.0002)$, but there was no significant additional contribution made to the variance in the parents' PHQ9 scores by the inclusion of the boys' Hyperactivity scores ( $R$ square change $=0.042$, $F(1,29)$ for change $=2.023, p=0.166$. The boys' Irritability scores showed only a trend towards making a significant unique contribution to the variance in their parents' GAD7 scores ( $\mathrm{R}$ squared change $=0.125, F(1,29)$ for change $=4.139$, $p=0.051$ ), and was not further examined. Boys' ages were controlled for in these regression analyses because of the abovementioned significant inverse correlation between boys' ages and their Hyperactivity and Irritability scores: age made no significant contribution to the boys' parents' PHQ9 scores ( $R$ square $=0.055$, $F(1,30)=1.702, \quad p=0.202)$, or to their GAD7 scores $(\mathrm{R}$ square $=0.090$, $F(1,30)=2.858, p=0.102)$. The autistic girls' Hyperactivity scores made a significant contribution to their parents' GAD7 scores (R squared change $=0.251, F(1,30)$

Table 2 Pearson correlation coefficients between autistic children's ABC subscale scores and parents' GAD7 and PHQ9 scores

\begin{tabular}{llllll}
\hline Parents & Boys & & & \\
\cline { 2 - 6 } & Irritability & Lethargy & Stereotypy & Hyperactivity & $\begin{array}{l}\text { Inap- } \\
\text { propriate } \\
\text { Speech }\end{array}$ \\
\hline GAD7 & $\mathbf{. 4 7 8 * *}^{*}$ & .240 & .006 & .353 & .088 \\
PHQ9 & $\mathbf{. 5 6 9 * * *}$ & .342 & .156 &. $\mathbf{4 7 0 * *}$ & .248 \\
& Girls & & & & .342 \\
GAD7 & .216 & .375 & .356 & $\mathbf{. 5 0 1 *}$ & .284 \\
PHQ9 & .334 &. $\mathbf{4 1 4}$ & .340 & .303 & \\
\hline
\end{tabular}

${ }^{*} p<.05, * * p<.01, * * * p<.001$ 
for change $=10.053, p=0.003)$, and their Lethargy scores made a significant contribution to their parents' PHQ9 scores ( $\mathrm{R}$ squared change $=0.171, F(1,30)$ for change $=6.208, p=0.018$ ). These differences in the patterns of associations between the ABC subscales and parental GAD7 or PHQ9 scores are despite the abovementioned lack of significant differences in the scores of the autistic boys and girls on these ABC subscales.

\section{ABC Item Level}

As mentioned in the Methods, these $\mathrm{ABC}$ subscale scores are based upon sets of multiple $\mathrm{ABC}$ items, almost all of which refer to discrete behaviours under the general category of "Challenging". Investigation of which specific ABC items were most strongly linked with parental GAD7 and PHQ9 scores might add detail to these findings and precision to clinical implications.

Because of the large number of ABC items in the Irritability (15 items), Lethargy (16 items), and Hyperactivity (16 items) subscales, an exploration of their association with parents' GAD7 and PHQ9 scores was undertaken firstly by Pearson Correlation coefficients. Taking the boys and their parents first, there were two 'large' effect coefficients between items drawn from the ABC Irritability subscale and parental PHQ9 scores. For the girls, there were five ABC Hyperactivity items that had correlations $>0.4$ (i.e., in the upper range of 'medium' effect sizes) with their parents' GAD7 score. The girls' parents' PHQ9 scores were significantly correlated at a medium effect strength with three of the girls' ABC Lethargy subscale items. These $10 \mathrm{ABC}$ items represent the subset that was most strongly associated with the boys' and girls' parents' GAD7 and PHQ9, and are shown in Table 3.

These particular ABC subscale items were compared for the contribution they make to parental GAD7 and PHQ9 scores by a series of hierarchical regression analyses, entering the particular ABC subscale items in order of the size of the correlation coefficients. It is relevant to note that there were no significant differences in any these $10 \mathrm{ABC}$ items according to the sex of the autistic child: MANOVA main effect $F(10,53)=1.692, p=0.107, \mu^{2}=0.242$; all univariate effects were $p>0.125$.

The boys' ABC Irritability subscale item "Mood changes quickly" made a significant contribution to their parents' PHQ9 score, R square change $=0.294, F(1,30)$ for change $=12.687, p=0.001$, but the second ABC item ("Has temper tantrums or outbursts when does not get his own way") did not significantly contribute to the variance in parents' PHQ9 scores, R squared change $=0.028, F$ for change $=1.240$, $p=0.275$. Age was entered first into this analysis but did not make a significant contribution to parents' PHQ9 scores ( $F$ for change $=1.070, p=0.275$ ).

When the five ABC Hyperactivity subscale items shown in Table 3 that had significant correlations with the girls' parents' GAD7 score were entered into hierarchical regression in order of their coefficient size, only the first of these ("Impulsive" [acts without thinking]") made a significant contribution to the variance in GAD7 scores, R squared change $=0.243, F(1,30)$ for change $=9.608, p=0.004$. The remaining four $\mathrm{ABC}$ items had $\mathrm{R}$ for change scores $<0.086, p>0.066$. 
Table 3 Medium and Large effect size Pearson correlation coefficients between autistic children's ABC subscale items and parents' GAD7 and PHQ9 scores

\begin{tabular}{|c|c|c|}
\hline Parents & Boys & $r$ \\
\hline PHQ9 & $\begin{array}{l}\text { ABC Irritability subscale items } \\
\text { "Mood changes quickly" } \\
\text { "Has temper tantrums or outbursts when does } \\
\text { get his own way" } \\
\text { Girls }\end{array}$ & $\begin{array}{l}.573 * * \\
.543 * *\end{array}$ \\
\hline GAD7 & $\begin{array}{l}\text { ABC Hyperactivity subscale items } \\
\text { "Impulsive (acts without thinking)" } \\
\text { "Pays no attention when spoken to" } \\
\text { "Restless, unable to sit still" } \\
\text { "Easily distractible" } \\
\text { "Disturbs others" }\end{array}$ & $\begin{array}{l}.493 * * \\
.464 * * \\
.456 * \\
.407 * \\
.406 *\end{array}$ \\
\hline PHQ9 & $\begin{array}{l}\text { ABC Lethargy subscale items } \\
\text { "Difficult to reach, contact, or get through to" } \\
\text { "Inactive, never moves spontaneously" } \\
\text { "Isolates herself from other children/adults" }\end{array}$ & $\begin{array}{l}.492 * * \\
.423 * \\
.402 *\end{array}$ \\
\hline
\end{tabular}

A similar result was found when the three ABC Lethargy subscale items were entered into a hierarchical regression on the girls' parents' PHQ9 score: only the first item ("Difficult to reach, contact, or get through to") made a significant contribution to variance in PHQ9 score, $\mathrm{R}$ squared change $=0.242, F(1,30)$ for change $=9.572$, $p=0.004$. The remaining two ABC Lethargy subscale items had $\mathrm{R}$ for change scores $<0.052, p>0.155$.

The three stages of this analysis of the effect of autistic boys' and girls' ABC data on parents' GAD7 and PHQ9 scores are depicted in Table 4, and indicate the extra levels of specificity regarding which aspects of $\mathrm{ABC}$-measured $\mathrm{CB}$ that were most powerfully associated with the GAD7 and PHQ9 scores shown by these autistic children's parents. Although those results suggest that there were significant associations between the girls' and boys' ABC total scores, selected subscale scores, and particular item scores, and their parents' GAD7 and PHQ9 scores, when these three levels of the ABC contributor variables were tested for their relative contributions to their parents' GAD7 and PHQ9 scores via hierarchical regression, that apparently shared contribution model was not confirmed. For boys, as shown above, the item "Mood changes quickly" made a significant contribution to parents' PHQ9 scores, but the ABC Hyperactivity subscale score and the ABC Total score (R square change $=0.008, p=0.572$ ) did not. For girls, the ABC item "Impulsive (acts without thinking)" made a significant contribution to their parents' GAD7 scores but the ABC Hyperactivity subscale score ( $\mathrm{R}$ square change $=0.005, p=0.647$ ) and $\mathrm{ABC}$ Total score $(\mathrm{R}$ square change $=0.008, p=0.580$ ) did not; the girls' scores on $\mathrm{ABC}$ item "Difficult to reach, contact, or get through to" made a significant contribution to their parents' PHQ9 scores, but the ABC Lethargy subscale score (R square change $=0.014, p=0.467)$ and $\mathrm{ABC}$ Total score ( $\mathrm{R}$ square change $=0.003$, $p=0.755$ ) did not. These results are depicted by the solid arrows shown in Table 4 . 
Table 4 ABC correlates and contributors to parents' GAD7 and PHQ9 scores

\begin{tabular}{|c|c|c|c|c|}
\hline \multicolumn{3}{|l|}{ Boys } & \multicolumn{2}{|l|}{ Parents $^{1}$} \\
\hline $\mathrm{ABC}$ & ABC Subscale & $\mathrm{ABC}$ items & GAD7 & PHQ9 \\
\hline \multirow[t]{3}{*}{ Total } & & & $r=.359$ & $r=.498$ \\
\hline & Irritability & & & $\mathrm{R}^{2}$ change $=.371$ \\
\hline & & "Mood changes quickly" & & $\mathrm{R}^{2}$ change $=.294$ \\
\hline \multicolumn{5}{|l|}{ Girls } \\
\hline \multirow[t]{5}{*}{ Total } & & & $r=.421$ & $r=.428$ \\
\hline & Hyperactivity & & $\mathrm{R}^{2}$ change $=.251$ & \\
\hline & & "Impulsive (acts without thinking)" & $\mathrm{R}^{2}$ change $=.243$ & \\
\hline & Lethargy & & & $\mathrm{R}^{2}$ change $=.171$ \\
\hline & & $\begin{array}{l}\text { "Difficult to reach, contact, or get through } \\
\text { to" }\end{array}$ & & $\mathrm{R}^{2}$ change $=.242$ \\
\hline
\end{tabular}

\section{Discussion}

Although there were no significant differences between the GAD7 and PHQ9 scores of the parents of autistic boys or autistic girls, Fig. 1 suggests that both sets of parents exhibited higher levels of mild, moderate and (to a lesser extent) severe anxiety than the general community sample reported by Stocker et al. (2021). Similarly, both sets of parents reported higher levels of mild and moderate depression, and (for the parents of autistic girls) moderately severe depression. Figure 1 suggests that the proportions of these parents who reported minimal anxiety or no depression were much smaller than those reported in the general community, suggestive of a subpopulation of parents of an autistic child who have elevated anxiety and depression compared to the general community. Direct comparisons between these findings and those from previous studies are of limited value due to the lack of adequate instrumentation for GAD and MDD in those studies, but these data extend those findings to the use of standardised DSM-5 based scales. Inclusion of GAD and MDD provides a more relevant index of parental mental health than measures of 'parental stress' and extends the findings of the anxiety-provoking and depressive effects of parenting an autistic boy (Bitsika et al., 2014) to include parents of autistic girls and boys who were matched for age, IQ and ASD severity.

The absence of any statistically significant differences in the presence or severity of the ABC total and subscale scores between autistic boys and girls also supports the comparability of these samples of autistic boys and girls (as well as age, IQ and ASD severity). With that in mind, the differences in the correlations between the ABC subscales and parental GAD7 and PHQ9 scores (Table 2) argue for the presence of different 'connections' between child CB and parental anxiety and depression across autistic girls and boys. Although no causal links can be drawn from these purely correlational data, the link between the autistic boys' Irritability and their parents' anxiety and depression were not present for the autistic girls and their parents. Similarly, although the autistic girls' Lethargy was significantly associated with their parents' depression, that was not the case for the autistic boys and their 
parents. Finally, Hyperactivity was significantly associated with the boys' parents' depression but not their anxiety, and the reverse association was found for the autistic girls and their parents.

However, it is when these subscale data were unpacked according to the actual $\mathrm{ABC}$ items representing specific $\mathrm{CBs}$ that the most interesting and valuable results were found for clinical settings. Although the boys' mood changes significantly contributed to their parents' depression, there was no significant additional contribution made to their parents' anxiety by the autistic boys' total ABC or Hyperactivity subscale scores. By contrast, it was the autistic girls' isolation (in terms of their parents' inability to reach, contact, or get through to their daughters) that contributed most to their parents' depression, and the girls' impulsivity that contributed most to their parents' anxiety. These specific challenging behaviours were the strongest contributors to the anxiety and depression reported by the parents of these autistic boys and girls, more powerful than either the global $\mathrm{ABC}$ score or any of the $\mathrm{ABC}$ subscale scores, as indicated by the solid arrows in Table 4. It is worthwhile to recall that there were no significant differences between the autistic boys' and girls' scores on any of these particular $\mathrm{ABC}$ items.

Thus, the task of parenting an autistic child brings with it substantially higher levels of anxiety and depression, but the precise linkages between specific CBs and parental anxiety and depression appear to be different between autistic boys and girls. Fast and unpredictable mood changes, impulsive behaviour, and social isolation, would test the fortitude of any parents, and the exacerbation and/or consistency of these behaviours appears to be a key factor in parental anxiety and depression when an autistic child is present. Thus, the first implication that these findings have for clinical settings is that parental anxiety and depression may be linked with different child CBs depending upon whether the autistic child is a boy or a girl.

Secondly, parents appeared to be more sensitive to their sons' or daughters' specific CBs, despite the actual (non-significantly different) severity of those CBs. This then moves the strength of these associations between child CBs and parental anxiety and depression from the child's exhibition of those behaviours to the parents' reaction to them. From these results, it appears that parents of an autistic boy are more likely to find his abrupt changes of mood depressive, but not be similarly bothered by his impulsivity or social isolation, whereas these latter CBs are most likely to engender anxiety and depression respectively in the parents of autistic girls. Because there were no significant differences in the severity of these particular ABC items across the autistic boys and girls, this finding speaks to differences in the sensitivity of parents to these particular CBs. Exactly why parents of an autistic child should react differently to the same levels of these CBs depending on the sex of their child is not immediately apparent from these data, and is a major focus for future research. The presence of social contingencies may lead to differing parental expectations for autistic girls' and boys' behaviour, but little is known about this issue at this time. The need to focus research onto autistic girls has been highlighted in the literature recently, but little mention has been made to date about the differences in parental GAD or MDD across autistic girls and boys. While it is possible that the exhibition of these parent-rated CBs may emphasise differences between their expectations for their autistic daughters and sons, these explanations are hypothetic 
at this stage. Similarly, the social engagement of autistic girls, even via 'camouflaging' behaviour (Hull et al., 2017), may suggest that these girls' CB are influenced in ways that boys' CB are not, perhaps flowing on to their parents' GAD and MDD.

Third, the provision of supportive therapy for parents of an autistic child is a major component of treatment models for autistic families. For example, when the autistic child is in preschool, helping parents understand their child's developmental progress, engage in interactive play, and facilitate language, have been reported as of major relevance to parents (Whitaker, 2002). Parents of autistic children of older age (up to 18 years) report that they need consistent therapies for their child, as well as for professionals who are knowledgeable about autism (Siklos \& Kerns, 2006). Although group therapy and support may be relevant for some parents of an autistic child (Bitsika \& Sharpley, 2000), there is also some evidence that individual treatment for the parent's anxiety and depression may also be of benefit (Blackledge \& Hayes, 2006). However, all of these treatment and support models are based upon the assumption that the autistic child causes the same kind of anxiety and depression among their parents, regardless of the sex of that child. The current data challenge that assumption, and suggest that different types of child CBs may be associated with parental anxiety and depression depending on the sex of the autistic child. Further, it may be that addressing parental expectations about their autistic child's CBs might be a worthwhile addition to the kinds of support and treatment that have been used to date.

Limitations of the study include a sample that was adequate but not very large, and so statistical power could be increased by a larger sample. The autistic boys and girls were all mildly impaired, and so no extension of these findings can be made to more severely impaired autistic children. The sample was from 6 to 18 years in age, and so there is a limitation upon applying these results to younger autistic children or adults. Geographical and cultural limitations are also present, and the lack of such comparisons across different nations and social groups hinders the generalisability of findings such as these. This issue is largely ignored in the ASD literature, and is a potential source of confound in methodology and disagreement in results. The preponderance of mothers over fathers restricts the ability to comment on sex of parent as a factor. Finally, these data are a 'snapshot' of the sample at a single point in time, and do not allow for examination of any fluctuations over time or age of the autistic child.

Some strengths of the study are the use of robust measures of parental anxiety and depression by including scales that assess the diagnostic criteria for GAD and MDD. The ABC is a well-validated and reliable scale that has been used in many studies of autistic children. The matching of the autistic boys and girls on age, IQ and ASD severity adds to the reliability of the comparative data across the girls and boys and their parents. Although not planned, the lack of any significant differences in the boys' and girls' ABC total, subscale, or the 10 selected ABC items, adds to the verisimilitude of the comparison made of the associations between children's CBs and parental anxiety and depression.

In conclusion, these findings extend the previous research by use of standardised measures of parental GAD and MDD. The finding that there appear to be different connections between specific CBs and parental anxiety and depression across 
autistic boys and girls argues for careful consideration of parental (unmet) expectations regarding the behaviour of their autistic child. This aspect of parenting an autistic child has yet to be extensively investigated, and may hold a key to understanding how parenting autistic children might be accomplished with least anxiety and depression.

Acknowledgements The authors thank the parents of these children for their participation in this study.

Funding Open Access funding enabled and organized by CAUL and its Member Institutions No funding was received for this research.

\section{Compliance with ethical standards}

Research involving human participants The study was approved by the Bond University Human Research Ethics Committee, constituted according to the Helsinki Declaration of 1964 and recent amendments, approval no. RO1516.

Informed consent Written informed consent to participate was provided by these parents for themselves and for their children, and the children themselves gave verbal assent.

Conflicts of interest Neither author has any conflict of interest to declare.

Open Access This article is licensed under a Creative Commons Attribution 4.0 International License, which permits use, sharing, adaptation, distribution and reproduction in any medium or format, as long as you give appropriate credit to the original author(s) and the source, provide a link to the Creative Commons licence, and indicate if changes were made. The images or other third party material in this article are included in the article's Creative Commons licence, unless indicated otherwise in a credit line to the material. If material is not included in the article's Creative Commons licence and your intended use is not permitted by statutory regulation or exceeds the permitted use, you will need to obtain permission directly from the copyright holder. To view a copy of this licence, visit http://creativecommons.org/ licenses/by/4.0/.

\section{References}

Aman, M., Singh, N., Stewart, A., \& Field, C. (1985). The Aberrant Behavior Checklist: A behavior ratings scale for the assessment of treatment effects. American Journal of Mental Deficiency, 89, $485-491$.

APA. (2000). Diagnostic and statistical manual of mental disorders (4th ed., Text Revision). American Psychiatric Association.

APA. (2013). Diagnostic and Statistical Manual of Mental Disorders-5th edition. American Psychiatric Association.

Ashworth, M., Palikara, O., \& Van Herwegen, J. (2019). Comparing parental stress of children with neurodevelopmental disorders: The case of Williams syndrome, Down syndrome and autism spectrum disorders. Journal of Applied Research in Intellectual Disabilities, 32(5), 1047-1057.

Bitsika, V., \& Sharpley, C. (2000). Development and testing of the effects of support groups on the well-being of parents of children with autism-II: Specific stress management strategies. Journal of Applied Health Behaviour, 2, 8-15.

Bitsika, V., \& Sharpley, C. (2016). Brain-Behaviour Research Group Autism Study.

Bitsika, V., Sharpley, C., Sweeney, J., \& McFarlane, J. (2014). HPA and SAM axis responses as correlates of self- vs parental ratings of anxiety in boys with an Autistic Disorder. Physiology \& Behaviour, 127, 1-7. 
Bitsika, V., \& Sharpley, C. F. (2004). Stress, anxiety and depression among parents of children with autism spectrum disorder. Australian Journal of Guidance \& Counselling, 14, 151-161.

Blackledge, J., \& Hayes, S. (2006, 2006/03/14). Using Acceptance and Commitment Training in the Support of Parents of Children Diagnosed with Autism. Child \& Family Behavior Therapy, 28(1), 1-18.

Cohen, J. (1988). Statistical power for the behavioural sciences. Erlbaun.

Dabrowska, A., \& Pisula, E. (2010). Parenting stress and coping styles in mothers and fathers of preschool children with autism and Down syndrome. Journal of Intellectual Disability Research, 54(3), 266-280.

Estes, A., Munson, G., Dawson, E., Koehler, E., Zhou, X.-H., \& Abbott, R. (2009). Parenting stress and psychological functioning among mothers of preschool children with autism and developmental delay. Autism, 13, 357-387.

Falk, N., Norris, K., \& Quinn, M. (2014). The Factors Predicting Stress, Anxiety and Depressionin the Parents of Children with Autism. Journal of Autism and Developmental Disabilities, 44, 3185-3203.

Fries, E., Hesse, J., Hellhammer, J., \& Hellhammer, D. (2005). A new view on hypocortisolism. Psychoneuroendocrinology, 30, 1010-1016.

Giovagnoli, G., Postorino, V., Fatta, L., Sanges, V., De Peppo, L., Vassena, L., Rose, P., Vicari, S., \& Mazzone, L. (2015, 2015/10/01/). Behavioral and emotional profile and parental stress in preschool children with autism spectrum disorder. Research in Developmental Disabilities, 45-46, 411-421.

Glidden, D., Bouman, W., Jones, B., \& Arcelus, J. (2016, 2016/01/01/). Gender Dysphoria and Autism Spectrum Disorder: A Systematic Review of the Literature. Sexual Medicine Reviews, 4(1), 3-14.

Hayes, S., \& Watson, S. (2013). The impact of parenting stress: A meta-analysis of studies comparing the experience of parenting stress in parents of children with and without Autism Spectrum Disorder. Journal of Autism and Developmental Disabilities, 43, 629-642.

Hull, L., Petrides, K., Allison, C., Smith, P., Baron-Cohen, S., Lai, M., \& Mandy, W. (2017). "Putting on my best normal": Social camouflaging in adults with autism spectrum conditions. Journal of Autism and Developmental Disorders, 47, 2519-2534.

Kaat, A., Lecavalier, L., \& Aman, M. (2014). Validity of the Aberrant Behaviour Checklist in children with Autism Spectrum Disorder. Journal of Autism and Developmental Disorders, 44, 1103-1116.

Kaplan, M., Asnis, G., Sanderson, W., Keswani, L., de Lecuona, J., \& Joseph, S. (1994). Suicide assessment: Clinical interview vs. self-report. Journal of Clinical Psychology, 50(2), 294-298.

Kroenke, K., Spitzer, R., \& Williams, J. (2001). The PHQ-9: Validity of a brief depression severity measure. Journal of General Internal Medicine, 16, 606-613.

Kuusikko, S., Pollock-Wurman, R., Jussila, K., Carter, A., Mattila, M.-L., Ebeling, H., Pauls, D., \& Moilanen, I. (2008). Social anxiety in high-functioning children and adolescents with Autism and Asperger Syndrome. Journal of Autism and Developmental Disorders, 38, 1697-1709.

Langa, K. M., Valenstein, M. A., Fendrick, A. M., Kabeto, M. U., \& Vijan, S. (2004). Extent Cost of Informal Caregiving for Older Americans With Symptoms of Depression. American Journal of Psychiatry, 161, 857-863.

Langewitz, W., \& Ruddell, H. (1989). Spectral analysis of heart rate variability under mental stress. Journal of Hypertension, 7, 32-33.

Lecavalier, L., Leone, S., \& Wiltz, J. (2006). The impact of behaviour problems on caregiver stress in young people with autism spectrum disorders. Journal of Intellectual Disability Research, 50(3), 172-183.

Lord, C., Rutter, M., DiLavore, P., Risi, S., Gotham, K., \& Bishop, S. (2012). Autism Diagnostic Observation Schedule, Second Edition (ADOS-2). Western Psychological Services.

Lovibond, S., \& Lovibond, P. (1995). Manual for the Depression Anxiety Stress Scales (2 ed.). Psychology Foundation.

Malone, K., Haas, G., Sweeney, J., \& Mann, J. (1995, 6/8/). Major depression and the risk of attempted suicide. Journal of Affective Disorders, 34(3), 173-185.

Moussavi, S., Chatterji, S., Verdes, E., Tandon, A., Patel, V., \& Ustun, B. (2007). Depression, chronic diseases, and decrements in health; results from the World Health Surveys. Lancet, 370, 851-858.

Mugno, D., Ruta, L., D’Arrigo, V., \& Mazzone, L. (2007). Impairment of quality of life in parents of children and adolescents with pervasive developmental disorder. Health and Quality of Life Outcomes, $5,22$.

Mykletun, A., Bjerkeset, O., Øverland, S., Prince, M., Dewey, M., \& Stewart, R. (2009). Levels of anxiety and depression as predictors of mortality: The HUNT study. British Journal of Psychiatry, 195, $118-125$. 
Nutt, D. (2004). Anxiety and depression: Individual entities or two sides of the same coin? International Journal of Psychiatry in Clinical Practice, 8, 19-24.

O'Nions, E., Happé, F., Evers, K., Boonen, H., \& Noens, I. (2018, 2018/04/01). How do Parents Manage Irritability, Challenging Behaviour, Non-Compliance and Anxiety in Children with Autism Spectrum Disorders? A Meta-Synthesis. Journal of Autism and Developmental Disorders, 48(4), $1272-1286$.

Rao, P., \& Beidel, D. (2009). The Impact of Children with High-Functioning Autism on Parental Stress, Sibling Adjustment, and Family Functioning. Behavior Modification, 33(4), 437-451. https://doi. org/10.1177/0145445509336427

Sharpley, C., Bitsika, V., \& Efremidis, B. (1997). Influence of gender, parental health and perceived expertise of assistance upon stress, anxiety and depression among parents of children with autism. Journal of Intellectual and Developmental Disability, 22, 19-28.

Shawler, P., \& Sullivan, M. (2017). Parental Stress, Discipline Strategies, and Child Behavior Problems in Families With Young Children With Autism Spectrum Disorders. Focus on Autism and Other Developmental Disabilities, 32(2), 142-151. https://doi.org/10.1177/1088357615610114

Siklos, S., \& Kerns, K. (2006, 2006/10/01). Assessing Need for Social Support in Parents of Children with Autism and Down Syndrome. Journal of Autism and Developmental Disorders, 36(7), 921-933.

Spitzer, R., Kroenke, K., Williams, J., Group, P. H. Q. P. C. S. (1999). Validation and Utility of a Selfreport Version of PRIME-MD: The PHQ Primary Care Study. Journal of the American Medical Association, 282(18), 1737-1744.

Spitzer, R., Kroenke, K., Williams, J., \& Lowe, B. (2006). A brief measure for assessing Generalised Anxiety Disorder. Archives of Internal Medicine, 166, 1092-1097.

Stocker, R., Tran, T., Hammarberg, K., Nguyen, H., Rowe, H., \& Fisher, J. (2021, 2021/04/01/). Patient Health Questionnaire 9 (PHQ-9) and General Anxiety Disorder 7 (GAD-7) data contributed by 13,829 respondents to a national survey about COVID-19 restrictions in Australia. Psychiatry Research, 298, 113792.

Tabachnik, B., \& Fidell, L. (2013). Using multivariate statistics (6th ed.). Pearson Education.

Tomanik, S., Harris, G., \& Hawkins, J. (2004). The relationship between behaviours exhibited by children with autism and maternal stress. Journal of Intellectual and Developmental Disability, 29, 16-26.

Wechsler, D. (2011). The Wechsler Abbreviated Scale of Intelligence (2nd ed). Pearson.

Whitaker, P. (2002). Supporting Families of Preschool Children with Autism: What Parents Want and what Helps. Autism, 6, 411-426.

Zimmerman, M., McDermut, W., \& Mattia, J. (2000). Frequency of anxiety disorders in psychiatric patients with Major Depressive Disorder. American Journal of Psychiatry, 157, 1337-1340.

Publisher's Note Springer Nature remains neutral with regard to jurisdictional claims in published maps and institutional affiliations. 